\title{
Elevated Fusiform Cell Activity in the Dorsal Cochlear Nucleus of Chinchillas with Psychophysical Evidence of Tinnitus
}

\author{
T. J. Brozoski, ${ }^{1}$ C. A. Bauer, ${ }^{1}$ and D. M. Caspary ${ }^{2}$ \\ ${ }^{1}$ Division of Otolaryngology, Head and Neck Surgery and ${ }^{2}$ Department of Pharmacology, Southern Illinois University \\ School of Medicine, Springfield, Illinois 62702
}

\begin{abstract}
Chinchillas with psychophysical evidence of chronic tinnitus were shown to have significantly elevated spontaneous activity and stimulus-evoked responses in putative fusiform cells of the dorsal cochlear nuclei (DCN). Chinchillas were psychophysically trained and tested before and after exposure to a traumatic unilateral $80 \mathrm{~dB}$ (sound pressure level) $4 \mathrm{kHz}$ tone. Before exposure, two groups were matched in terms of auditory discrimination performance (noise, and 1, 4, 6, and $10 \mathrm{kHz}$ tones). After exposure, a single psychophysical difference emerged between groups. The exposed group displayed enhanced discrimination of $1 \mathrm{kHz}$ tones ( $p=0.00027$ ). Postexposure discrimination of other stimuli was unaffected. It was hypothesized that exposed animals experienced a chronic subjective tone (i.e., tinnitus), resulting from their trauma, and that features of this subjective tone were similar enough to $1 \mathrm{kHz}$ to affect discrimination of $1 \mathrm{kHz}$ objective signals. After psychophysical
\end{abstract}

testing, single-unit recordings were obtained from each animal's DCN fusiform cell layer. Putative fusiform cells of exposed animals showed significantly ( $p=0.0136$ ) elevated spontaneous activity, compared with cells of unexposed animals. Putative fusiform cells of exposed animals showed a greater stimulus-evoked response to tones at $1 \mathrm{kHz}(p=0.0000006)$ and at characteristic-frequency ( $p=0.0000009)$. This increased activity was more pronounced on the exposed side. No increase in stimulus-evoked responses was observed to other frequencies or noise. These parallel psychophysical and electrophysiological results are consistent with the hypothesis that chronic tonal tinnitus is associated with, and may result from, trauma-induced elevation of activity of DCN fusiform cells.

Key words: tinnitus; acoustic trauma; psychophysical animal model; dorsal cochlear nucleus; fusiform cells; neural plasticity
Thirty-five percent of the population of the United States experiences tinnitus, a subjective "ringing in the ears" (US, 1967); $10 \%$ of these consider their symptoms to be severe and disabling (Cooper, 1994). The most common causes of tinnitus are agerelated hearing loss and hearing loss secondary to noise trauma. Noise-induced hearing loss typically becomes evident in middle age, and the associated tinnitus is present for a significant portion of an individual's life. Despite the prevalence and morbidity of tinnitus, the pathophysiology of the disorder is poorly understood, and there is no generally accepted cure or treatment. This lack of understanding stems from the difficulty of studying a perceptual state with no objective stimulus correlate and that tinnitus may arise from diverse combinations of peripheral and central pathologies (Murai et al., 1992).

Following the work of Jastreboff et al. (1988), we developed a psychophysical animal model of tinnitus to investigate the disorder in controlled experiments, where the cause is known and the population sample is homogeneous (Bauer and Brozoski, 2001). A conditioned-suppression method was used to quantify the sensation of tinnitus in rats (Jastreboff et al., 1988). Unilateral acoustic trauma was used to induce tinnitus, and continuous freeoperant lever pressing for food reinforcement was used to

\footnotetext{
Received Dec. 28, 2001; revised Dec. 28, 2001; accepted Jan. 2, 2002.

This work was supported by National Institutes of Health Grants DC96-003 and DC00-151. We thank Andrew Feltovich for assistance in data analysis.

Correspondence should be addressed to T. J. Brozoski, Department of Surgery, Division of Otolaryngology, Head and Neck Surgery, 801 North Rutledge, Southern Illinois University School of Medicine, Springfield, IL 62702. E-mail: tbrozoski@siumed.edu.

Copyright (ㄷ) 2002 Society for Neuroscience $\quad 0270-6474 / 02 / 222383-08 \$ 15.00 / 0$
}

measure chronic tinnitus over weeks to months. Using this model in rats, tinnitus can be psychophysically distinguished from simple hearing loss, and the tinnitus can be partially reversed using a GABA analog (Bauer and Brozoski, 2001). This model can also be used to measure qualitative aspects of tinnitus.

Recent studies suggest that tinnitus may result from inappropriate plastic changes in the auditory pathway in response to acoustic trauma (Potashner et al., 1997; Kaltenbach et al., 2000; Milbrandt et al., 2000). Consistent with this hypothesis is evidence that aberrant neural activity in the dorsal cochlear nucleus (DCN) might underlie the generation of tinnitus. In patients suffering from somatic craniocervical tinnitus, Levine (1999) hypothesized the likely involvement of the DCN. Zhang and Kaltenbach (1998) and Kaltenbach and Afman (2000) have measured elevated multiunit activity in the DCN of rats and hamsters after acoustic trauma. They observed that the spontaneous DCN hyperactivity evoked by previous noise exposure was similar to the response to low-to-moderate-intensity tone stimuli. Melamed et al. (2000) reported that selective outer hair cell loss induced by cisplatin resulted in increased spontaneous DCN activity. The preceding studies established that abnormal DCN activity occurred in subjects after acoustic trauma but did not establish a connection between tinnitus and abnormal activity in the DCN. The present controlled experiments were designed to investigate this association using chinchillas, a widely studied animal model of human auditory processing (Clark, 1991). Fusiform cells were studied because there is evidence for plasticity in this cell type after noise exposure and in aging (Kaltenbach et al., 1998; Schatteman et al., 2000). Fusiform cells form the primary output of the DCN projecting via the dorsal acoustic stria to the contralateral 
inferior colliculus (Osen, 1972; Romand and Avan, 1997; Alibardi, 2000).

\section{MATERIALS AND METHODS}

Subjects. Twelve, young adult male chinchillas (Moulton Chinchilla Farm, Rochester, MN), were individually housed and maintained at $25^{\circ} \mathrm{C}$ with a $12 \mathrm{hr}$ reversed light/dark schedule. Complete data were obtained from eight animals: two animals died before completion of the study, and two were eliminated from the study because of poor behavioral performance in initial training. Subjects were maintained on a restricted diet with body weight at $\sim 80 \%$ of free feeding weight. The experimental protocol was approved by the Southern Illinois University School of Medicine Laboratory Animal Care and Use Committee, and the experiment was conducted in accordance with the Policy for Use of Animals in Research established by the Society for Neuroscience.

Psychophysical training and testing. Subjects were trained and tested using established behavioral techniques described in previous studies (Brozoski et al., 1996; Bauer et al., 1998; Bauer et al., 1999; Bauer and Brozoski, 2001). Before exposure to acoustic trauma, chinchillas were trained to lever press for food in individual commercial operant conditioning chambers (model 80200; Lafayette Instruments, Lafayette, IN) that were located in a double-wall sound-attenuation chamber. There was no visual contact between subjects once inside the conditioning chambers.

Data collection and behavioral contingencies were independently controlled for each subject. Stimulus conditions and behavioral contingencies were computer-controlled using custom software. Subjects were trained and tested in daily $60 \mathrm{~min}$ sessions. After subjects met performance criteria for stable lever pressing, a variable interval reinforcement schedule was imposed. Reinforcement schedules were individualized for each subject to maximize performance before acoustic trauma. Once established, all schedules remained the same throughout testing. Criteria for stable operant performance are described below. Acoustic stimuli were digitally synthesized (SRS DS345; Stanford Research Systems, Sunnyvale, CA) and transduced (Realistic speaker, model 40-1398; Radio Shack, Fort Worth, TX) using a speaker that was center-mounted in the lid of each conditioning chamber. Continuous broad-band noise $(\mathrm{BBN})$ at $60 \mathrm{~dB}$ sound pressure level (SPL) was present in each chamber, except during test stimulus presentations. Test stimuli were presented to subjects in synchrony.

Auditory test stimuli were presented in pseudorandomly scheduled 60 sec test periods within each session. Lever-pressing activity in response to the auditory test stimuli was used to derive auditory discrimination functions. Test stimuli were chosen to extend over the frequency range of greatest sensitivity for the chinchilla: $1,4,6$, and $10 \mathrm{kHz}$ tones. The frequency of the test stimulus was fixed within a given session, and randomly varied across sessions. Each test stimulus was presented at five different intensities within each session. The presentation order of each stimulus intensity was randomized and repeated once for a total of 10 $(5 \times 2)$ stimulus presentations in a session. The range of intensity levels varied with the stimulus frequency but always included $0 \mathrm{~dB}$ SPL and extended from near hearing threshold to clearly above threshold.

In each session a suppression ratio $(R)$, a running relative performance index, was calculated for successive 1 min periods: $R=B / A+B$, where $B$ is the number of lever presses in the current period, and $A$ is the number of lever presses in the immediately preceding period. $R$ provided a running index of behavior that enabled a quantitative comparison of behavior during test stimulus periods to behavior during baseline noise. When the lever-press rate is constant, $R=0.5$. As the lever-press rates drop below baseline, $R$ decreases, and as lever-press rates rise above baseline, $R$ increases. Before psychophysical functions can be derived from $R$, it is necessary to establish a contingency between operant performance and auditory sensation level. This was done through suppression training. In suppression training, and throughout testing, if a subject's $R$ during a $0 \mathrm{~dB}$ presentation was $>0.2$, they were given a $0.5 \mathrm{~mA}$ foot shock, $1 \mathrm{sec}$ in duration, at the conclusion of the $0 \mathrm{~dB}$ period. This contingency trained subjects to stop lever pressing $(R \approx 0)$ when no objective sound $(0 \mathrm{~dB})$ was present. During test presentations of stimuli with intensities of $>0 \mathrm{~dB}$, subjects were free to lever press for food without foot shock. Three criteria had to be met for individual-subject data to be included in further analysis: (1) There had to be a minimum of 200 lever presses in the session; (2) mean $R$ for background noise periods (i.e., baseline performance) had to be $>0.4$, with (3) a SD of $<0.2$. These criteria were empirically chosen to improve measurement reliability: sessions with 200 or more responses had, on average, >3

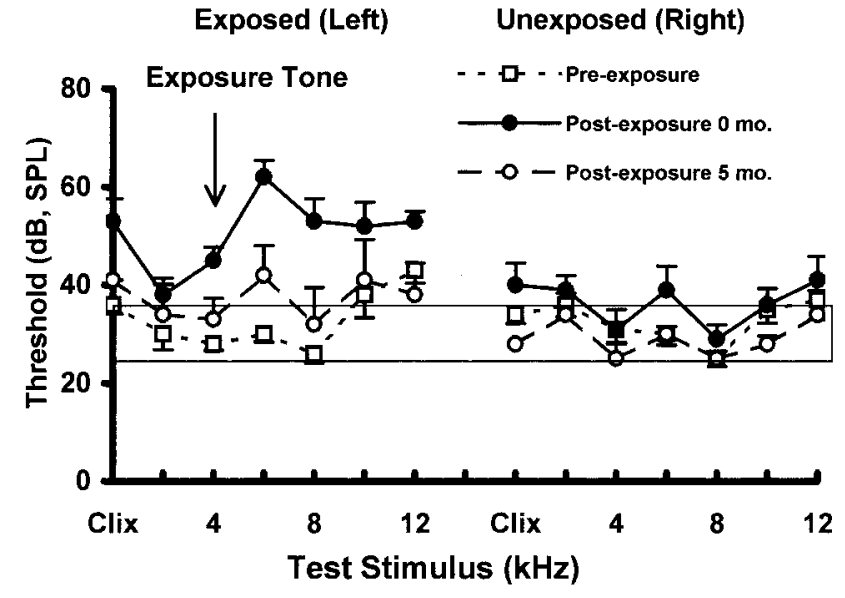

Figure 1. Auditory thresholds before and after trauma. Thresholds were measured using acoustically evoked brainstem potentials, recorded immediately after stimulus onset. Shown are the mean threshold functions (error bars indicate $1 \mathrm{SEM})$ for trauma-exposed subjects $(n=4)$. The normative range was determined by the average, \pm 1 SEM, of four unexposed control animals. The trauma stimulus is indicated by the arrow. Immediately after exposure there was a significant ipsilateral threshold elevation (left panel), compared with pre-exposure levels, for tones from 4 to $6 \mathrm{kHz}(p=0.005-0.007)$ and for clicks $(p=0.01)$ in the exposed ear (ipsilateral). There was no significant threshold elevation for $1 \mathrm{kHz}$ tones immediately after trauma $(p=0.08)$, nor were there significant threshold elevations 5 months after trauma for any stimulus $(p=0.157-0.719)$.

presses per minute, thereby permitting suppression to be adequately measured in successive 1 min periods; a mean $R>0.4$ for baseline performance permitted some minute-to-minute baseline variation but eliminated sessions with more than a few minutes of nonresponding; finally, sessions with extremely variable response rates were eliminated by the SD criterion. Stimulus discrimination functions were derived from $R$ measured during test stimulus presentations. Data from a minimum of five criterion-level test sessions, for each stimulus, were averaged to derive individual and group discrimination functions for each stimulus: BBN, and $1,4,6,10 \mathrm{kHz}$ tones.

After psychophysical data were collected from each subject, for each of the auditory test stimuli, subjects were anesthetized, acoustic brainstem response (ABR) thresholds were determined, and half the subjects were exposed to unilateral acoustic trauma. One week after recovery from anesthesia, all subjects resumed psychophysical testing. Post-trauma psychophysical functions were obtained for the same test stimuli (BBN, and $1,46,10 \mathrm{kHz}$ tones) following the protocol described. It is important to note that training subjects before acoustic trauma, i.e., as in the present design, produces different psychophysical consequences than those obtained when training subjects after acoustic trauma (see Bauer and Brozoski (2001) and Discussion, "Tinnitus versus hyperacusis").

Acoustic trauma. After initial psychophysical training and testing, the animals were divided into two matched groups, of four animals each (group 1: control; group 2: acoustic trauma), equated for psychophysical discrimination performance. Animals were anesthetized with an intramuscular (IM) ketamine $\mathrm{HCl}$-xylazine $(34 / 4 \mathrm{mg} / \mathrm{kg}$ ) mixture and placed in a modified stereotaxic head frame. Subjects in group 2 were unilaterally exposed to a $4 \mathrm{kHz} 80 \mathrm{~dB}$ SPL tone using a $3 \mathrm{~mm}$ cone-shaped speculum mounted to a high-frequency speaker (model 40-1398, Realistic; Radio Shack) inserted into the left external auditory canal. Exposure durations of 30-60 min were sufficient to elevate ABR thresholds 20-30 dB (SPL) in the left ear (Fig. 1). Contralateral ABR thresholds were not elevated because of interaural attenuation and effective containment of the sound field by the insert speculum. Trauma was induced using a moderate level of sound intensity $(80 \mathrm{~dB})$ to limit the extent of cochlear damage caused by the exposure. Nevertheless, because subjects in the present study were exposed while anesthetized and would not have had a functional acoustic reflex, their cochlear damage would be expected to be greater than that obtained with free field exposure in unanesthetized subjects. There are no published studies of threshold shifts in chinchillas or humans using the present method of acoustic trauma. Salvi et al. (1978), however, reported 15-20 dB permanent 
threshold shifts in chinchillas exposed to octave band noise at $86 \mathrm{~dB}$ SPL in a free field for $5 \mathrm{~d}$.

ABR thresholds were determined in each ear before and after acoustic trauma inside a double-wall sound-attenuation chamber using an Intelligent Hearing Systems (Miami, FL) high-frequency system. Subdermal stainless steel recording electrodes were inserted posterior to each pinna, with a reference electrode located at dorsal cranial midline and a ground electrode located in a rear leg. ABR thresholds were obtained for clicks and tone bursts, $5 \mathrm{msec}$ in duration, presented at a rate of $50 / \mathrm{sec}$. Tone bursts were gated using an exact Blackman envelope ( $2.5 \mathrm{msec}$ rise/decay, $0 \mathrm{msec}$ plateau). Evoked potentials were averaged over 1024 sweeps. Amplifier gain was $200 \mathrm{k}$ and filtered using a $100-3000 \mathrm{~Hz}$ bandpass. Recording epochs comprised the $12 \mathrm{msec}$ after stimulus onset.

Electrophysiology. At the conclusion of post-trauma psychophysical testing, subjects in both groups were prepared for single-unit recordings from DCN neurons. Animals were anesthetized with a ketamine-xylazine mixture (described above), and rectal temperature was maintained at $37^{\circ} \mathrm{C}$ by a homeothermic blanket. Areflexia was assessed by absent response to a tail-pinch, and anesthesia was maintained by subsequent intramuscular administration of ketamine $(44 \mathrm{mg} / \mathrm{kg})$ alternated with ketamine-xylazine mix (8.5:1) at regular intervals (1-1.5 hr). ABRs were obtained immediately before surgery (Fig. 1, 5 months after exposure). Animals were placed in a stereotaxic instrument, a longitudinal incision was made at the midline behind the ears, neck muscles were reflected, and part of the occipital bone superior to the foramen magnum was removed to gain entry to the posterior fossa (Caspary et al., 1987).

Recording procedures were similar to those described by Palombi and Caspary (1996). Glass micropipettes filled with 2 м potassium acetate were beveled to tip resistances of $10-20 \mathrm{M} \Omega$ and advanced using a piezoelectric micropositioner (model 6000 ULN; Burleigh Instruments, Victor, NY) into first the left or right DCN. Care was taken to alternate starting recording from the left or right DCN across subjects. Signals were recorded via a silver-chloride silver wire coupled to the head stage of a preamplifier (model 8100; Dagan Instruments, Minneapolis, MN). Spikes were discriminated (model 120; World Precision Instruments, Sarasota, FL) with spike times recorded using an event timer (ET-1; Tucker-Davis Instruments, Sarasota, FL) set at $1.0 \mu \mathrm{sec}$ resolution and saved to computer disk for later analysis.

Acoustic stimuli under software control were presented monaurally via specially designed chinchilla ear bars to the ear ipsilateral to the DCN being examined. Ear bars were coupled to a DT48A earphone (Beyerdynamic, Farmingdale, NY), and a flexible probe microphone (Etymotic, Elk Grove Village, IL) tube placed near the tympanum. This probe was used to monitor the stimulus and generate calibration tables in decibels of SPL (with respect to $20 \mu$ pa) for use by programmable attenuators. Acoustically driven units were located using 5 msec clicks, 60-80 dB (SPL). Post-stimulus time histograms (PSTHs) for temporal classification were generated from responses to 200 presentations of characteristic frequency (CF) tone bursts, $30 \mathrm{~dB}$ above $\mathrm{CF}$ threshold, $50 \mathrm{msec}$ duration, $5 \mathrm{msec}$ rise-fall (cosine) ramp, presented at a $5 / \mathrm{sec}$ rate. Spike times relative to signal onset were recorded and placed into bins (1500 bins, $0.075 \mathrm{msec}$ bin width, clock resolution $10 \mu \mathrm{sec}$ ). Spontaneous activity measurements were collected as 200 samples of $200 \mathrm{msec}$ bins. Units displaying prominent variability in spontaneous activity had spontaneous rates averaged over widely dispersed repeated (2-6) measurements. Complete data sets were obtained from units judged to be fusiform cells based on previously published criteria (Caspary, 1972; Godfrey et al., 1975; Rhode et al., 1983; Caspary et al., 1994; Backoff et al., 1997). These criteria included the following: (1) records obtained from units between 150 and $350 \mu \mathrm{m}$ into the DCN, as determined by the signal marker produced by puncturing the overlying pia mater, and onset of a characteristic click-evoked slow-wave; (2) units displaying buildup, pauserbuildup, or broad chopper responses; (3) units with large broad triphasic spikes. In most cases the electrode track could be followed through the fusiform cell layer, with termination of the track shortly beyond the layer boundary. Because of the curved shape of the DCN and the angle of approach, which was not perpendicular to the DCN dorsal surface, it was possible to enter the fusiform layer and, when the puncture was near the bend in the DCN, to record from several putative fusiform cells. On rare occasions electrode tracks exited the fusiform cell layer and re-entered at the lateral extreme. Successful electrode penetrations in each animal were marked with horseradish peroxidase (HRP) (4\%, type VI; Sigma,
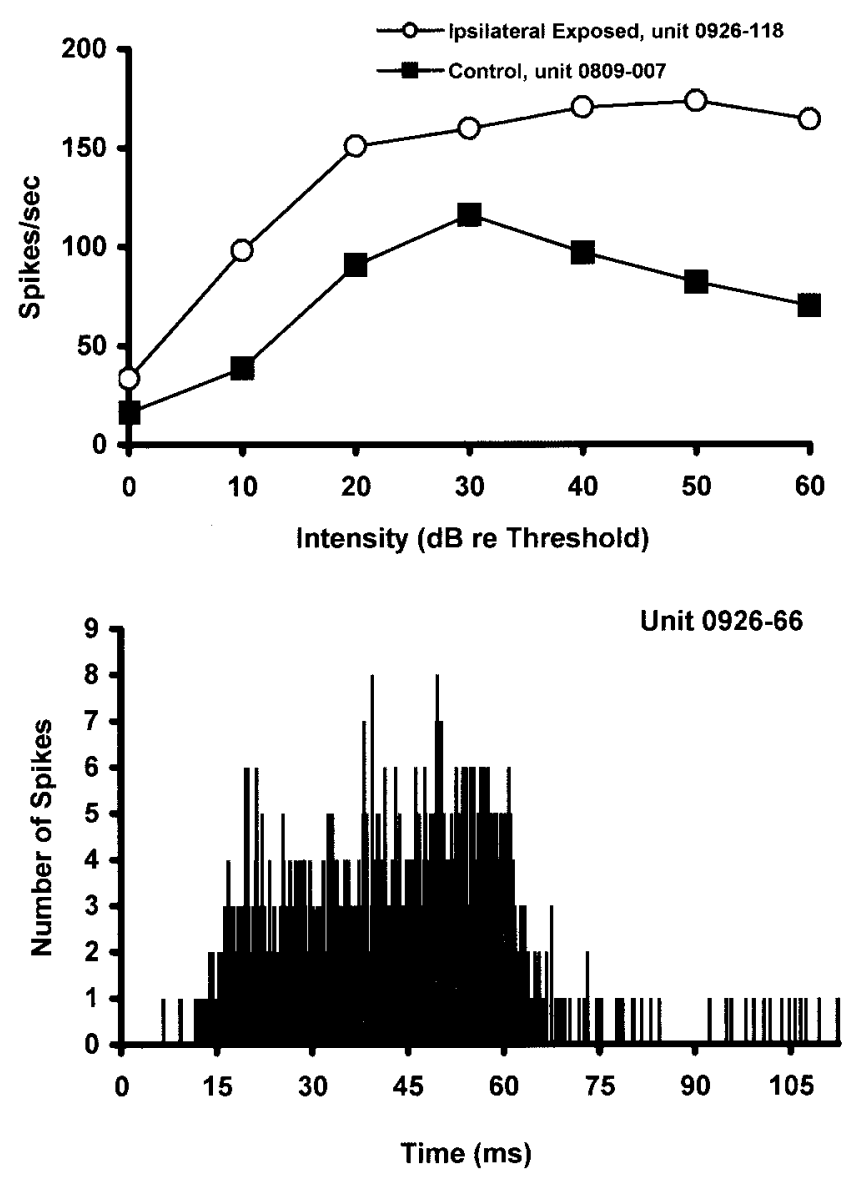

Figure 2. Representative PSTH and RIFs recorded from putative fusiform cells in the chinchilla DCN. Stimuli were presented to the ipsilateral ear. Top panel, RIFs of a unit recorded from the ipsilateral DCN of a trauma-exposed subject and a unit recorded from the DCN of an unexposed control subject. Bottom panel, Poststimulus histogram of a "buildup" unit from the ipsilateral DCN of a trauma-exposed subject. These profiles are similar to those reported by others for DCN fusiform units (Young, 1980).

St. Louis, MO) for histological confirmation of recording sites. At the conclusion of electrophysiological recording, animals were given an overdose of anesthetic and were perfused transcardially with $0.9 \%$ normal saline followed by $2.5 \%$ glutaraldehyde in $0.1 \mathrm{M}$ phosphate buffer. The brain was removed, placed in increasing concentrations of sucrose, and frozen sectioned using a sliding microtome. Sections were stained for HRP by incubation for $5 \mathrm{~min}$ at room temperature in $0.06 \%$ diaminobenzidine $\mathrm{HCl}$ (Sigma) and mounted on slides for confirmation of recording sites using phase-contrast light microscopy (Palombi and Caspary, 1996).

At the conclusion of the experiment, response characteristics of all units were reviewed, and a final designation of unit type was made off-line using the published criteria noted above, while blinded with respect to sound-exposed (ipsilateral, contralateral) versus control conditions. Representative DCN fusiform-like cell characteristics appear in Figure 2. Units were divided into two categories: putative fusiform and nonf usiform of unspecified subtype. Data were further analyzed within each classification. Descriptive statistics, graphic depictions, and inferential statistics were computed using spreadsheets (Excel; Microsoft Corp, Redmond, WA) with statistical enhancements (NAG, Inc., Downers Grove, IL). ASCII data files, stored during the experiments, were retrieved and directly imported into Excel spreadsheets. Psychophysical group differences were analyzed using mixed ANOVAs run on subjectby-subject averages at each stimulus intensity, thus insuring that each subject contributed equally to the analysis. Single-unit group differences were analyzed using the $t$ statistic; unequal variance was assumed, and exact $p$ values were determined. 


\section{RESULTS}

\section{Psychophysics}

A significant psychophysical difference between control and trauma subjects occurred only in the post-trauma discrimination of $1 \mathrm{kHz}$ tones. Pre-trauma and post-trauma discrimination functions are depicted in Figure 3, and the associated statistical analysis is summarized in Table 1 (mixed ANOVAs by stimulus type; main effects: treatment groups and stimulus intensity). There were no post-trauma psychophysical differences between control and trauma subjects when tested with 4,6 , and $10 \mathrm{kHz}$ tones or BBN. The post-trauma $1 \mathrm{kHz}$ discrimination function of the exposed group was shifted up relative to controls ( $p=$ 0.00027) (Table 1). An upward shift in discrimination functions reflects greater responding to the test stimuli (i.e., less suppression, or greater $R$ ). The upward shift of the $1 \mathrm{kHz}$ function of animals exposed to unilateral acoustic trauma indicates enhanced discrimination. This frequency-specific shift in the psychophysical discrimination function did not correspond to the frequency of maximum threshold shift measured in the ABR (Fig. 1). The maximum ABR threshold elevation, i.e., decreased sensitivity, was observed in the exposed ear at $6 \mathrm{kHz}$ (pre-trauma, $30 \pm 1.58$ $\mathrm{dB}$ vs post-trauma, $62 \pm 3.39 \mathrm{~dB}$ ). ABR thresholds at $1 \mathrm{kHz}$ were in the normal range for both ipsilateral (pre-trauma, $30 \pm 3.16$ $\mathrm{dB}$, vs post-trauma $33.8 \pm 3.39 \mathrm{~dB}$ ) and contralateral (pre-trauma, $36 \pm 2.45 \mathrm{~dB}$ vs post-trauma, $39 \pm 2.92 \mathrm{~dB}$ ) ears. At 5 months after trauma, hearing thresholds at all frequencies for both ears were at or near pre-trauma levels (Fig. 1, 5 months).

\section{Electrophysiology}

Data from 134 auditory units were obtained from subjects in both groups at the completion of psychophysical data collection, $\sim 5$ months after the single unilateral exposure to acoustic trauma at $4 \mathrm{kHz}$. Of these, $75 \mathrm{U}$ were classified as putative DCN fusiform cells (control left, $n=14$; control right, $n=17$; trauma ipsilateral, $n=27$; trauma contralateral, $n=17$ ), and 59 were classified as nonfusiform cells of unspecified subtype. Histologically confirmed HRP marks of recording sites from putative fusiform cells were all within the fusiform cell layer.

The spontaneous activity of putative fusiform cells was significantly elevated bilaterally in the DCN of chinchillas exposed to acoustic trauma compared with controls (Fig. 4$)(F=6.40$, df $=$ $1,74, p=0.0136)$. There was no significant difference between ipsilateral and contralateral cells of the trauma group, nor was there a significant difference between the left and right cells of the control group.

The dynamic range and slope of the rate-intensity functions (RIFs) of putative fusiform cells in trauma animals was significantly elevated at $1 \mathrm{kHz}$ compared with that of controls (Fig. 5). The effect was bilateral, but much more pronounced for ipsilateral cells (all intensities, re controls, $t=5.074$; $\mathrm{df}=342 ; p=$ 0.0000006 ), than contralateral cells (all intensities, re controls, $t=$ 2.60; $\mathrm{df}=198 ; p=0.010$ ). A similar significant RIF elevation was obtained at the CF of the neurons (ipsilateral all intensities re controls, $t=4.998$, df $=395, p=0.00000087$; contralateral re controls, $t=1.616$, df $=230, p=0.107)$. At other frequencies tested, there were no significant RIF differences between trauma (ipsilateral or contralateral) and control putative fusiform cells (Fig. 6). The absolute threshold of the cells was unaffected by trauma (measured at $\mathrm{CF}$, trauma ipsilateral vs control; $p=0.995$ ). However, trauma significantly increased the mid-range (10-40 $\mathrm{dB}$, re threshold) slope of the $1 \mathrm{kHz}$ RIF (trauma ipsilateral slope $=1.69$ vs control slope $=0.81 ; t=2.491 ; \mathrm{df}=43 ; p=0.017)$ as well as the maximum spike rate at $1 \mathrm{kHz}$ (trauma ipsilateral max. $=105.7$ vs control $\max =73.6 ; t=2.318 ; \mathrm{df}=49 ; p=$ $0.025)$. The effect of acoustic trauma on putative fusiform cell intensity encoding was, therefore, to increase the dynamic range of the response of the cell to particular stimuli.

It should be noted that the RIF results parallel the psychophysical results: a significant difference between the trauma and nontrauma discrimination functions was obtained only at $1 \mathrm{kHz}$; similarly, a significant difference between trauma and non-trauma RIFs was obtained at $1 \mathrm{kHz}$. RIF and discrimination functions for other stimuli were not significantly different between the groups, with the exception of CF RIFs, which have no psychophysical equivalent. Furthermore, there was a positive rank-order correlation between the psychophysical evidence of tinnitus $(R)$ in the trauma animals, and the maximum spike rate to $1 \mathrm{kHz}$ of putative fusiform cells recorded from those animals $(\rho=+0.60)$.

Electrophysiological evidence supporting a tinnitus hypothesis was confined to the putative DCN fusiform cells. There were no significant electrophysiological differences between control and trauma cells classified as nonfusiform.

\section{DISCUSSION}

The physiological basis of tinnitus is unknown. Most current theories of tinnitus (Tonndorf, 1987; Jastreboff, 1990; Penner and Bilger, 1995; Romand and Avan, 1997; Levine, 1999; Kaltenbach and Afman, 2000) invoke basic mechanisms of cochlear and auditory brainstem neurophysiology and do not require the presence of "higher order" cognition. On this basis there is no a priori reason animals cannot experience tinnitus. Nonverbal subjects should be quite capable of perceiving what is likely to be a primitive sensation.

\section{Central factors in tinnitus}

Despite the likely importance of peripheral factors, central processes must also significantly contribute to the sensation of tinnitus (Burns, 1984; Meikle, 1995). For example, human imaging studies have indicated widespread central activation during tinnitus episodes (Lockwood et al., 1998). It is not uncommon for humans to experience profound tinnitus after cochlear deafferentation either from ablation of the end organ or after bilateral eighth nerve transection (Anari et al., 1999). Although more than one brain area may contribute to the generation of tinnitus, a variety of circumstantial evidence points to the DCN as an area of considerable relevance. Tinnitus in humans often results from acoustic trauma (Loeb and Smith, 1967). In animals, acoustic trauma has been associated with a variety of pathological changes in the DCN (Caspary et al., 2001). Zhang and Kaltenbach (1998) and Kaltenbach and Afman (2000) reported increased DCN multiunit activity in rats and hamsters after acoustic trauma. Conditions other than trauma that commonly produce tinnitus in humans, such as salicylate ototoxicity, have been shown to affect GABA activity in the inferior colliculus of rats (Bauer et al., 2000); the inferior colliculus being a primary target of DCN fusiform cells (Beyerl, 1978).

\section{DCN activity and tinnitus}

Using an animal model, the present study suggests a direct connection between chronic tinnitus, as indicated by psychophysical measurement, and significantly elevated output of the DCN, as indicated by increased spontaneous activity and increased, frequency-specific, responses of cells displaying fusiform-like properties. Chronic tinnitus was induced by unilateral acoustic 

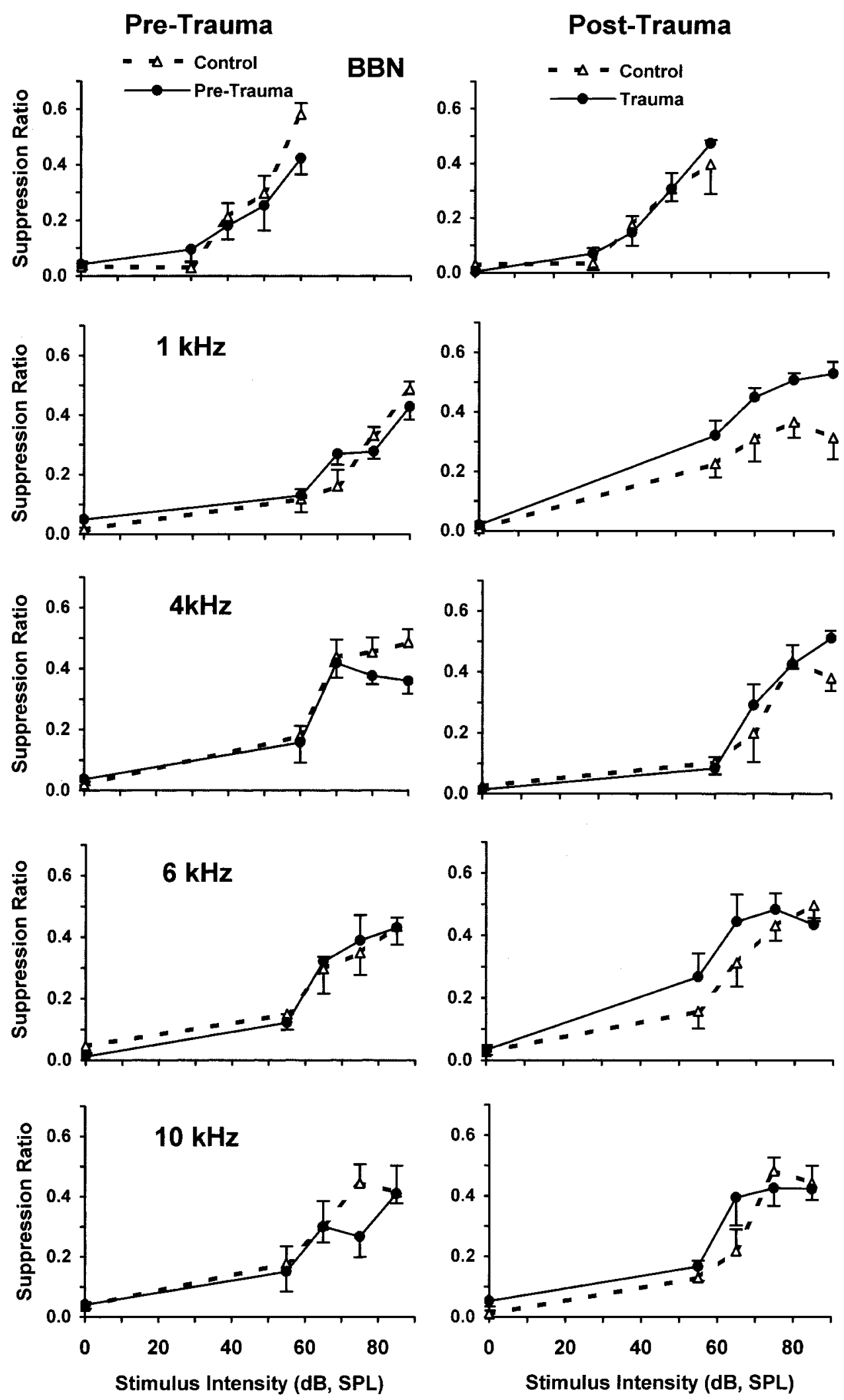

Figure 3. Pre-trauma versus posttrauma psychophysical discrimination functions (error bars indicate 1 SEM). Pre-trauma (left panels) and posttrauma (right panels) psychophysical discrimination functions for control (no trauma) and trauma-exposed subjects ( $n=4$ /group) are shown. Test stimulus values are indicated in each panel. Suppression ratios reflect the discrimination performance of subjects, with a value of 0 indicating discrimination of 0 $\mathrm{dB}$, and a value of 0.5 indicating discrimination of sound comparable to that of $60 \mathrm{~dB}$ broadband noise. The only significant difference between control (dashed lines) and trauma-exposed (solid lines) groups was for $1 \mathrm{kHz}$ tones after trauma (see Table 1 for significance levels).

trauma, i.e., a single exposure to a $4 \mathrm{kHz}$ tone at $80 \mathrm{~dB}$ (SPL), which produced a moderate temporary ipsilateral threshold elevation. Acoustic trauma in humans is commonly associated with tonal tinnitus (Loeb and Smith, 1967; Atherley et al., 1968). In the present study, chinchillas exposed to acoustic trauma showed a significant bilateral elevation of putative DCN fusiform cell spontaneous activity. Because the axons of DCN fusiform cells provide the major ascending output of the DCN (Osen, 1972; Young, 1980; Romand and Avan, 1997), increased spontaneous activity would be expected to affect higher auditory centers and could 
Table 1. Performance across all stimulus intensities: control group $(n=4)$ versus trauma group $(n=4)$

\begin{tabular}{|c|c|c|c|c|c|c|c|c|c|c|}
\hline & \multicolumn{5}{|c|}{ Pre-trauma } & \multicolumn{5}{|c|}{ Post-trauma } \\
\hline & $S S$ & df & $M S$ & $F$ & $p$ & $S S$ & $\mathrm{df}$ & $M S$ & $F$ & $p$ \\
\hline Noise & 0.010163 & 1,30 & 0.010163 & 1.02171 & 0.320196 & 0.001401 & 1,30 & 0.001401 & 0.12278 & 0.728487 \\
\hline $1 \mathrm{kHz}$ & 0.000771 & 1,30 & 0.000771 & 0.13565 & 0.715226 & 0.146065 & 1,30 & 0.146065 & 17.04054 & 0.000268 \\
\hline $4 \mathrm{kHz}$ & 0.020733 & 1,30 & 0.020733 & 2.85069 & 0.101705 & 0.013232 & 1,30 & 0.013232 & 1.39137 & 0.247444 \\
\hline $6 \mathrm{kHz}$ & 0.000001 & 1,30 & 0.000001 & 0.00014 & 0.990766 & 0.022891 & 1,30 & 0.022891 & 1.89077 & 0.179298 \\
\hline $10 \mathrm{kHz}$ & 0.017922 & 1,30 & 0.017922 & 1.30033 & 0.263176 & 0.012776 & 1,30 & 0.012776 & 1.09234 & 0.304302 \\
\hline
\end{tabular}

serve as the basis of the subjective sound sensation perceived as tinnitus. These findings support and extend the results of Kaltenbach et al. (1998), which described increased multiunit activity in the DCN after acoustic trauma (Kaltenbach et al., 1998; Zhang and Kaltenbach, 1998; Kaltenbach and Afman, 2000). In the present study, not only were the putative DCN fusiform cells shown to have significantly increased spontaneous activity after trauma, but also enhanced evoked responses to stimuli at the frequency for which there was psychophysical evidence of tinnitus.

\section{Bilateral DCN effects after unilateral noise exposure}

In the present study there were bilateral changes in the response properties of putative fusiform DCN neurons. Neurochemical and immunocytochemical data indicate the likelihood of crossed inhibitory connections between the cochlear nuclei, which appear to be glycinergic (Cant and Gaston, 1982; Wenthold, 1987; Schofield and Cant, 1996; Alibardi, 2000). Cant and Gaston (1982) reported connections between the dorsal and ventral cochlear nuclei projecting to the contralateral, anteroventral, and posteroventral cochlear nucleus, as well as to the DCN fusiform layer. Schofield and Cant (1996) described labeled boutons that made contacts in the contralateral fusiform cell and deep layers of the DCN. Potashner et al. (2000) found that unilateral manipulation of peripheral input altered glycine uptake, release, and strychnine binding in both the contralateral and ipsilateral DCN. Plastic changes in one cochlear nucleus are therefore likely to effect either direct or indirect changes in the contralateral cochlear nucleus. Furthermore, it is common for humans with unilateral hearing loss to experience tinnitus as a balanced bilateral sensation, i.e., a "center of the head" phenomenon.

\section{Tinnitus versus hearing loss}

It is important to note that the frequency-specific psychophysical and electrophysiological consequences of unilateral trauma at 4 $\mathrm{kHz}$ did not parallel the transient ABR threshold shifts caused by the trauma. In the present study, both psychophysical and singleunit electrophysiological data indicate a maximum effect of acoustic trauma at $1 \mathrm{kHz}$, considerably lower than the frequency of the trauma stimulus. When acute tinnitus is experimentally induced in human subjects using acoustic trauma, the resulting tinnitus is typically tonal, although its pitch has been reported to be quite variable. When the trauma stimulus was a pure tone, tinnitus frequency tended to be higher than the trauma frequency (Loeb and Smith, 1967), whereas 1/3-octave band noise produced tinnitus that was of lower frequency than the trauma stimulus (Atherley et al., 1968). In the present study, the maximum temporary unilateral threshold elevation, caused by ipsilateral trauma at 4 $\mathrm{kHz}$, appeared between 6 and $8 \mathrm{kHz}$ (Fig. 1). A similar pattern of hearing loss after acoustic trauma has been reported in chinchillas

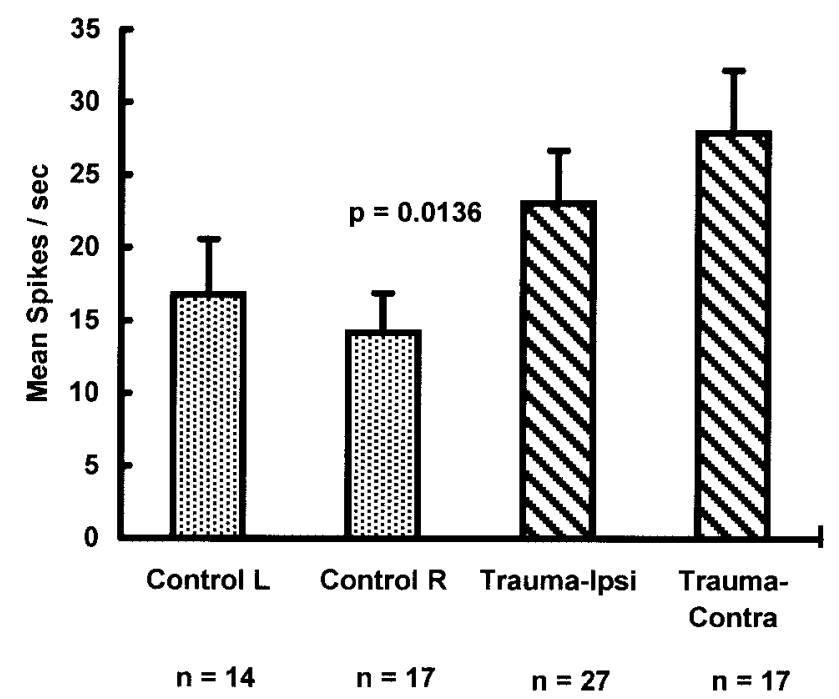

Figure 4. DCN fusiform cell spontaneous activity. Spontaneous activity of DCN fusiform cells in control and trauma-exposed chinchillas $(n=4$ animals/group). Spontaneous activity was measured in single units as cumulative spikes over $40 \mathrm{sec}$ epochs. Spontaneous activity is indicated as mean spikes per second (error bars indicate 1 SEM). Control data are presented for cells from the left and right DCN. Trauma data are presented for cells from the ipsilateral (left/exposed ear) and contralateral (right/unexposed ear) DCN. The number of units in each classification is shown below the $x$-axis. There was a significant difference in spontaneous activity between trauma fusiform cells (Trauma-Ipsi and Trauma-Contra) and control fusiform cells (Control $L$ and Control $R$ ), with no significant hemilateral differences.

(Salvi et al., 1982) and in humans (Ward, 1973). The psychophysical and electrophysiological results of the present study therefore correspond to results reported by other laboratories. The present results can be parsimoniously explained by the presence of tinnitus: the auditory discrimination of subjects in the trauma group was affected by a trauma-induced chronic tinnitus with characteristics similar to that of a $1 \mathrm{kHz}$ tone. Because subjects were trained to discriminate between the presence of an auditory signal and absence of a signal (0 dB SPL), when a signal was detected, subjects responded and did not suppress. More discriminable signals elevate responding toward the baseline $R$ value of 0.5 . It seems reasonable to assume that subjects with an internal signal (tinnitus) matching an external signal respond to the combined signals. The consequence would be an upward shift of the discrimination function for the objective signal matching the tinnitus. In the present study, that shift was at $1 \mathrm{kHz}$ (Fig. 3). Putative DCN fusiform cells reflected this as well with an increase in the dynamic range of their intensity response to $1 \mathrm{kHz}$ stimuli (Fig. 5). 

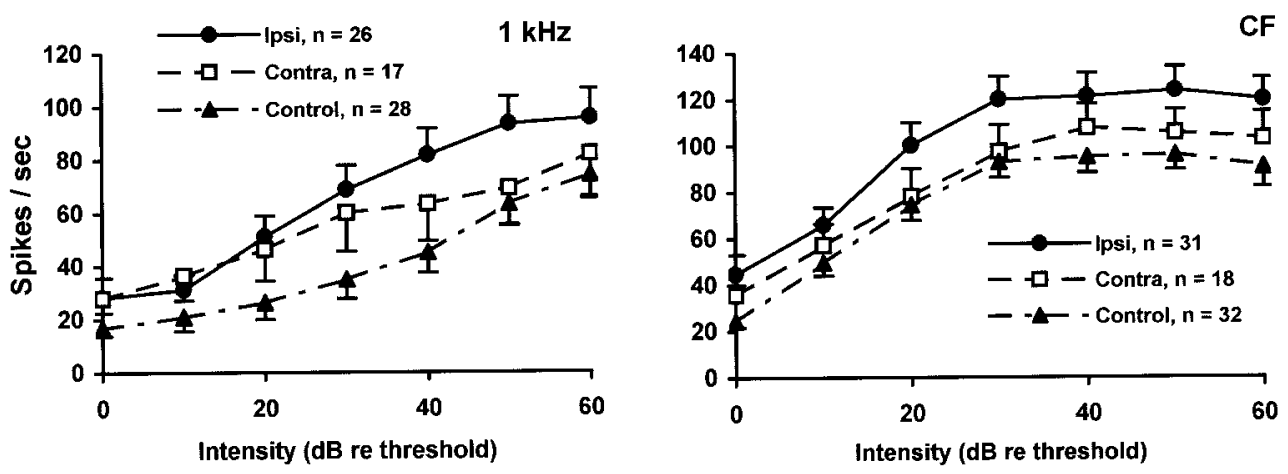

Figure 5. RIFs for $1 \mathrm{kHz}$ (left panel) and CF (right panel) tones. RIFs are shown for putative fusiform cells from the ipsilateral (Ipsi) and contralateral (Contra) DCN of trauma-exposed animals, as well as controls (left and right combined; error bars indicate 1 SEM). The $1 \mathrm{kHz}$ RIF of ipsilateral cells and contra cells was significantly elevated above that of controls, with the ipsilateral effect much more pronounced than the contralateral effect. The CF RIF of ipsilateral cells was significantly elevated above that of control cells as well as that of contralateral cells. The CF RIF of contra cells were not significantly different than that of controls. The number of cells contributing to each function is shown in the key. These electrophysiological results parallel the psychophysical results depicted in Figure 3.
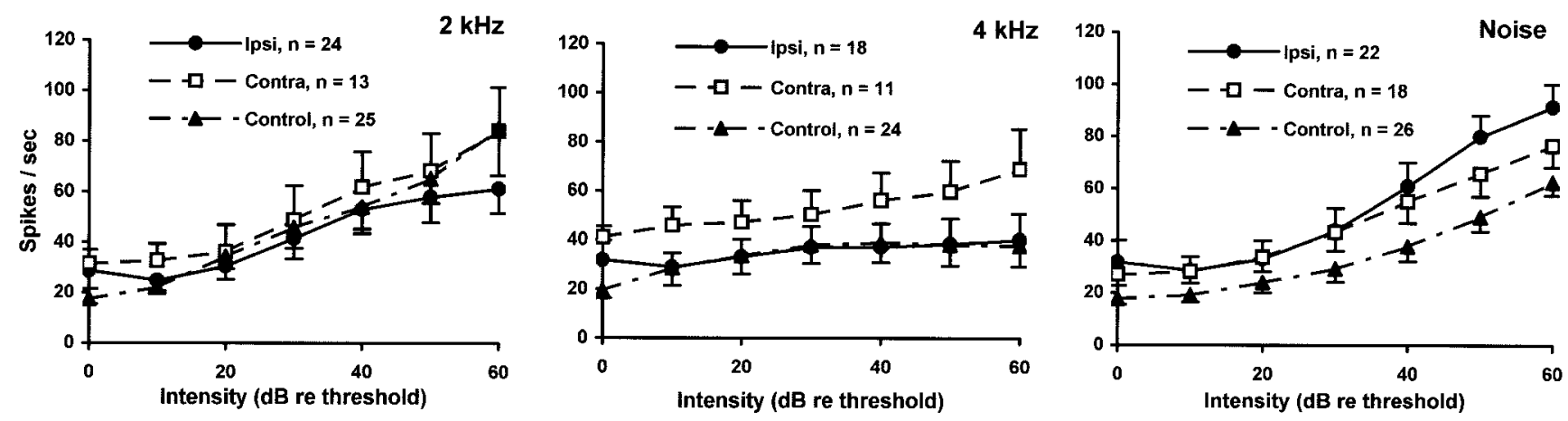

Figure 6. RIFs for stimuli other than $1 \mathrm{kHz}$ tones. RIFs are shown for putative fusiform cells from the ipsilateral (Ipsi) and contralateral (Contra) DCN of trauma-exposed animals, as well as controls (left and right combined; error bars indicate $1 \mathrm{SEM}$ ). There were no significant differences between the ipsilateral, contralateral, and control functions. The number of cells contributing to each function is shown in the key.

\section{Tinnitus versus hyperacusis}

Does the upward shift of the $1 \mathrm{kHz}$ discrimination functions in the trauma-exposed chinchillas reflect tinnitus or hyperacusis without tinnitus? Tinnitus in humans is often accompanied by hyperacusis (US, 1967). However, hyperacusis in humans is typically not restricted to a single frequency, and the hyperacusis of tinnitus patients is not restricted to the frequency match of their tinnitus (Anari et al., 1999). In the present experiment, the psychophysical consequence of trauma appeared at a single frequency, unlike typical hyperacusis. Furthermore, it has been shown that changing the design of the animal experiments, inducing tinnitus before initial training (Jastreboff et al., 1988; Bauer and Brozoski, 2001), downshifts the discrimination functions. In this alternative paradigm, tinnitus serves as a signal for suppression rather than responding (whereas in the present design silence served as the signal for suppression). Therefore, hyperacusis alone does not provide a satisfactory explanation of the present results.

\section{DCN mechanisms and tinnitus}

Chronic tinnitus may result from loss of glycinergic inhibition within the DCN stemming from a trauma-induced loss of DCN afferent input (Caspary et al., 2001). Results in the present study are consistent with a loss of glycinergic inhibition onto fusiform cells in the DCN. The observed shift in the RIF is similar to observations of iontophoretic blockade of glycine receptors onto fusiform cells (Davis and Young, 2000). Potashner et al. (2000) reported "a long-lasting decline in the electrically evoked release of $\left[{ }^{14} \mathrm{C}\right]$ Gly" bilaterally in the DCN of guinea pigs after unilateral cochlear ablation. This loss of inhibitory function in the DCN may increase DCN output above pretrauma levels. The increase in afferent auditory activity could result in the sensation of tinnitus.

Many experiments have shown that cochlear trauma results in decreased activity of primary afferents to the cochlear nuclei (Salvi et al., 1982; Dancer et al., 1992). Paradoxically and consistent with the present hypothesis, Boettcher and Salvi (1993) reported increased ventral cochlear neural responses in PST histograms after a 3-5 min pure tone exposure in chinchilla neurons displaying inhibition. Recent studies report age-related shifts in RIFs, similar to changes found in the present study, recorded from rat fusiform cells (Schatteman et al., 2000). A variety of experiments have shown that cochlear trauma results in increased neural activity at various loci along the central auditory pathway (Willott and Lu, 1981; Salvi et al., 1990; Szczepaniak and Moller, 1996; Wang et al., 1996; Syka and Rybalko, 2000). The present experiment extends those findings by establishing a connection between moderate acoustic trauma, increased putative fusiform cell activity in the DCN, and chronic tinnitus. Future experi- 
ments will have to demonstrate that augmented DCN fusiform cell activity is necessary for the expression of tinnitus.

\section{REFERENCES}

Alibardi L (2000) Cytology of large neurons in the guinea pig dorsal cochlear nucleus contacting the inferior colliculus. Eur J Histochem 44:365-375.

Anari M, Axelsson A, Eliasson A, Magnusson L (1999) Hypersensitivity to sound-questionnaire data, audiometry and classification. Scand Audiol 28:219-230.

Atherley GRC, Hempstock TI, Noble WG (1968) Study of tinnitus induced temporarily by noise. J Acoust Soc Am 44:1503-1506.

Backoff PM, Palombi PS, Caspary DM (1997) Glycinergic and GABAergic inputs affect short-term suppression in the cochlear nucleus. Hear Res 110:155-163.

Bauer CA, Brozoski TJ (2001) Assessing tinnitus and prospective tinnitus therapeutics using a psychophysical animal model. J Assoc Res Otolaryngol 2:54-64.

Bauer CA, Herr LA, Brozoski TJ (1998) Behavioral evidence of chronic tinnitus in rats with unilateral noise trauma. Neuroscience Abstr 24:1634.

Bauer CA, Brozoski TJ, Rojas R, Boley J, Wyder M (1999) Behavioral model of chronic tinnitus in rats. Otolaryngol Head Neck Surg 121:457-462.

Bauer CA, Brozoski TJ, Holder TM, Caspary DM (2000) Effects of chronic salicylate on GABAergic activity in rat inferior colliculus. Hear Res 147:175-182.

Beyerl BD (1978) Afferent projections to the central nucleus of the inferior colliculus in the rat. Brain Res 145:209-223.

Boettcher FA, Salvi RJ (1993) Functional changes in the ventral cochlear nucleus following acute acoustic overstimulation. J Acoust Soc Am 94:2123-2134.

Brozoski TJ, Wyder M, Bauer CA (1996) An animal model of tinnitus that reflects some of the qualitative features and the persistence of the disorder. Neurosci Abstr 22:1821.

Burns EM (1984) A comparison of variability among measurements of subjective tinnitus and objective stimuli. Audiology 23:426-440.

Cant NB, Gaston KC (1982) Pathways connecting the right and left cochlear nuclei. J Comp Neurol 212:313-326.

Caspary DM (1972) Classification of subpopulations of neurons in the cochlear nuclei of the kangaroo rat. Exp Neurol 37:131-151.

Caspary DM, Pazara KE, Kossl M, Faingold CL (1987) Strychnine alters the fusiform cell output from the dorsal cochlear nucleus. Brain Res 417:273-282.

Caspary DM, Backoff PM, Finlayson PG, Palombi PS (1994) Inhibitory inputs modulate discharge rate within frequency receptive fields of anteroventral cochlear nucleus. J Neurophysiol 72:2124-2133.

Caspary DM, Salvi RJ, Helfert RH, Brozoski TJ, Bauer CA (2001) Neuropharmacology of noise induced hearing loss in brainstem auditory structures. In: Noise induced hearing loss: mechanisms of damage and means of prevention (Henderson D, Prasher D, Kopke R, Salvi RJ, Hamernik R, eds), pp 169-186. London: NRN.

Clark WW (1991) Recent studies of temporary threshold shift (TTS) and permanent threshold shift (PTS) in animals. J Acoust Soc Am 90:155-163.

Cooper Jr JC (1994) Health and Nutrition Examination Survey of 197175: Part II. Tinnitus, subjective hearing loss, and well-being. J Am Acad Audiol 5:37-43

Dancer AL, Henderson D, Salvi RJ, Hamernik RP (1992) Noiseinduced hearing loss. St. Louis: Mosby.

Davis KA, Young ED (2000) Pharmacological evidence of inhibitory and disinhibitory neuronal circuits in dorsal cochlear nucleus. J Neurophysiol 83:926-940.

Godfrey DA, Kiang NY, Norris BE (1975) Single unit activity in the dorsal cochlear nucleus of the cat. J Comp Neurol 162:269-284.

Jastreboff PJ (1990) Phantom auditory perception (tinnitus): mechanisms of generation and perception. Neurosci Res 8:221-254.

Jastreboff PJ, Brennan JF, Coleman JK, Sasaki CT (1988) Phantom auditory sensation in rats: an animal model for tinnitus. Behav Neurosci 102:811-822.

Kaltenbach JA, Afman CE (2000) Hyperactivity in the dorsal cochlear nucleus after intense sound exposure and its resemblance to toneevoked activity: a physiological model for tinnitus. Hear Res 140:165-172.

Kaltenbach JA, Godfrey DA, Neumann JB, McCaslin DL, Afman CE, Zhang J (1998) Changes in spontaneous neural activity in the dorsal cochlear nucleus following exposure to intense sound: relation to threshold shift. Hear Res 124:78-84.

Kaltenbach JA, Zhang J, Afman CE (2000) Plasticity of spontaneous neural activity in the dorsal cochlear nucleus after intense sound exposure. Hear Res 147:282-292.

Levine RA (1999) Somatic (craniocervical) tinnitus and the dorsal cochlear nucleus hypothesis. Am J Otolaryngol 20:351-362.
Lockwood AH, Salvi RJ, Coad ML, Towsley ML, Wack DS, Murphy BW (1998) The functional neuroanatomy of tinnitus: evidence for limbic system links and neural plasticity. Neurology 50:114-120.

Loeb M, Smith RP (1967) Relation of induced tinnitus to physical characteristics of the inducing stimuli. J Acoust Soc Am 42:453-455.

Meikle MB (1995) The interaction of central and peripheral mechanisms in tinnitus. In: Mechanisms of tinnitus (Vernon JA, Moller AR, eds), pp 181-206. Needham Heights, MA: Allyn \& Bacon.

Melamed SB, Kaltenbach JA, Church MW, Burgio DL, Afman CE (2000) Cisplatin-induced increases in spontaneous neural activity in the dorsal cochlear nucleus and associated outer hair cell loss. Audiology 39:24-29.

Milbrandt JC, Holder TM, Wilson MC, Salvi RJ, Caspary DM (2000) GAD levels, muscimol binding in rat inferior colliculus following acoustic trauma. Hear Res 147:251-260.

Murai K, Tyler RS, Harker LA, Stouffer JL (1992) Review of pharmacologic treatment of tinnitus. Am J Otol 13:454-464.

Osen KK (1972) Projection of the cochlear nuclei on the inferior colliculus in the cat. J Comp Neurol 144:355-372.

Palombi PS, Caspary DM (1996) GABA inputs control discharge rate primarily within frequency receptive fields of inferior colliculus neurons. J Neurophysiol 75:2211-2219.

Penner MJ, Bilger RC (1995) Psychophysical observations and the origin of tinnitus. In: Mechanisms of tinnitus (Vernon JA, Moller AR, ed), pp 219-230. Needham Heights, MA: Allyn \& Bacon.

Potashner SJ, Suneja SK, Benson CG (1997) Regulation of D-aspartate release and uptake in adult brain stem auditory nuclei after unilatera middle ear ossicle removal and cochlear ablation. Exp Neurol 148:222-235.

Potashner SJ, Suneja SK, Benson CG (2000) Altered glycinergic synaptic activities in guinea pig brain stem auditory nuclei after unilateral cochlear ablation. Hear Res 147:125-136.

Rhode WS, Oertel D, Smith PH (1983) Physiological response properties of cells labeled intracellularly with horseradish peroxidase in cat ventral cochlear nucleus. J Comp Neurol 213:448-463.

Romand R, Avan P (1997) Anatomical and functional aspect of the cochlear nucleus. In: The central auditory system. (Ehret G, Romand R, eds), pp 97-191. New York: Oxford UP.

Salvi RJ, Hamernik RP, Henderson D (1978) Discharge patterns in the cochlear nucleus of the chinchilla following noise induced asymptotic threshold shift. Exp Brain Res 32:301-320.

Salvi R, Perry J, Hamerink RP, Henderson D (1982) Relationships between cochlear pathologies and auditory nerve and behavioral responses following acoustic trauma. In: New perspective on noiseinduced hearing loss (Hamerink RP, Henderson D, Salvi R, eds), pp 165-188. New York: Raven.

Salvi RJ, Saunders SS, Gratton MA, Arehole S, Powers N (1990) Enhanced evoked response amplitudes in the inferior colliculus of the chinchilla following acoustic trauma. Hear Res 50:245-257.

Schatteman TS, Hughes LF, Caspary DM (2000) Evidence of an agerelated loss of inhibitory function in rat DCN output neurons: rate responses. Soc Neurosci Abstr 30:957.

Schofield BR, Cant NB (1996) Origins and targets of commissural connections between the cochlear nuclei in guinea pigs. J Comp Neurol 375:128-146.

Syka J, Rybalko N (2000) Threshold shifts and enhancement of cortical evoked responses after noise exposure in rats. Hear Res 139:59-68.

Szczepaniak WS, Moller AR (1996) Evidence of neuronal plasticity within the inferior colliculus after noise exposure: a study of evoked potentials in the rat. Electroencephalogr Clin Neurophysiol 100:158-164.

Tonndorf J (1987) The analogy between tinnitus and pain: a suggestion for a physiological basis of chronic tinnitus. Hear Res 28:271-275.

United States National Center for Health Statistics (1967) Characteristics of persons with impaired hearing, United States, July 1962-June 1963; demographic and other characteristics of persons with a binaural hearing impairment, classified according to amount of hearing loss. Washington, DC: US Government Printing Office.

Wang J, Salvi RJ, Powers N (1996) Plasticity of response properties of inferior colliculus neurons following acute cochlear damage. J Neurophysiol 75:171-183.

Ward WD (1973) Modern Developments in Audiology. In: Modern developments in audiology, Ed 2 (Jerger JF, ed), pp 301-344. New York: Academic.

Wenthold RJ (1987) Evidence for a glycinergic pathway connecting the two cochlear nuclei: an immunocytochemical and retrograde transport study. Brain Res 415:183-187.

Willott JF, Lu S-M (1981) Noise-induced hearing loss can alter neural coding and increase excitability in the central nervous system. Science 216:1331-1332.

Young ED (1980) Identification of response properties of ascending axons from dorsal cochlear nucleus. Brain Res 200:23-37.

Zhang JS, Kaltenbach JA (1998) Increases in spontaneous activity in the dorsal cochlear nucleus of the rat following exposure to high-intensity sound. Neurosci Lett [Erratum (1998) 252:668] 250:197-200. 